\title{
Temperature Effects on the Propagation Characteristics of Love Waves along Multi-Guide Layers of $\mathrm{Sio}_{2} / \mathrm{Su}-8$ on St-90 ${ }^{\circ}$ X Quartz
}

Fangqian $\mathrm{Xu}^{1}$, Wen Wang ${ }^{2, *}$, Jiaoli Hou ${ }^{2}$ and Minghua Liu ${ }^{2}$

1 Zhejiang University of Media and Communications, Hangzhou 310018, China;

E-Mail: xufangqian2005@163.com

2 Institute of Acoustics, Chinese Academy of Sciences, Beijing 100190, China;

E-Mails: hou720@yahoo.com.cn (J.H.); liuminghua@mail.ioa.ac.cn (M.L.)

* Author to whom correspondence should be addressed; E-Mail: wangwenwq@mail.ioa.ac.cn; Tel.: +86-10-8254-7803; Fax: +86-10-8254-7802.

Received: 6 April 2012; in revised form: 21 May 2012 / Accepted: 22 May 2012 /

Published: 30 May 2012

\begin{abstract}
Theoretical calculations have been performed on the temperature effects on the propagation characteristics of Love waves in layered structures by solving the coupled electromechanical field equations, and the optimal design parameters were extracted for temperature stability improvement. Based on the theoretical analysis, excellent temperature coefficient of frequency ( $T c f$ ) of the fabricated Love wave devices with guide layers of $\mathrm{SU}-8 / \mathrm{SiO}_{2}$ on $\mathrm{ST}-90^{\circ} \mathrm{X}$ quartz substrate is evaluated experimentally as only $2.16 \mathrm{ppm}$.
\end{abstract}

Keywords: Love wave; multi-guide; $\mathrm{SU}-8 ; \mathrm{SiO}_{2} ; \mathrm{ST}-90^{\circ} \mathrm{X}$ quartz; $T c f$

\section{Introduction}

Recently, there has been great interest in Love wave devices in bio or chemical sensors owing to their very low longitudinal coupling attenuation, higher mass loading sensitivity and effective interdigital transducers (IDTs) protection in harsh gas and liquid environments [1-5]. Typical Love wave devices are composed of a piezoelectric substrate with an IDT pattern supporting a shear horizontal surface acoustic wave (SH-SAW), and a thin waveguide layer on the top of the substrate. Due to the waveguide effect, the acoustic energy is trapped into the thin guide layer, resulting in larger mass loading effects from any applied perturbation. One of the conditions for Love wave formation is 
the SH-SAW with high shear velocity propagating along the piezoelectric substrates. Another condition for the existence of Love wave mode is that the shear velocity in the guiding layer is smaller than the shear velocity in the substrate. As the difference of the shear velocities between the substrate and guiding layer becomes larger, the conversion efficiency of acoustic energy into the Love wave is increased, resulting in higher sensitivity to applied perturbations.

Various dielectric materials such as silicon dioxide $\left(\mathrm{SiO}_{2}\right)$ and polymers can be used as the waveguide materials [6]. $\mathrm{SiO}_{2}$ has been widely used for Love wave sensors because it presents the advantages of good rigidity, low acoustic loss, and high mechanical and chemical resistance. Nevertheless, the polymers have some advantages over $\mathrm{SiO}_{2}$ for Love wave sensor implementation because they are more efficient than $\mathrm{SiO}_{2}$ in converting the bulk $\mathrm{SH}$ mode to the Love wave mode due to their lower shear bulk velocity and lower density as compared to that of $\mathrm{SiO}_{2}$, resulting in an order of magnitude improvement in mass sensitivity [7]. Also, they are easier to deposit onto the substrate than $\mathrm{SiO}_{2}$.

Recently, a new Love wave structure containing both $\mathrm{SiO}_{2}$ and polymer films as a multilayer waveguide was proposed, and some promising results in the form of higher mass loading sensitivity and superior temperature stability were reported. Du et al. presented a new Love wave structure consisting of PMMA/SiO2/ST-quartz, which aims to utilize the merits of both PMMA and $\mathrm{SiO}_{2}$ and improve the overall performance of the devices $[8,9]$. Using the reversed polarity of the $T c f$ values of the materials for guide layer and substrate listed in Table 1, it is possible to realize a significant reduction in the temperature coefficients of the hybrid device, resulting in improvement of the temperature stability. The $\mathrm{SiO}_{2}$ thin film was frequently reported as the over-layer on $\mathrm{LiNbO}_{3}$ substrates to improve the temperature stability due to its reverse polarity temperature coefficient for the substrate, and related theoretical calculation and experiment were presented in some literatures [10-13]. However, to our knowledge, despite some meaningful performance of Love waves in multi-layered structures, there is still no systematic theoretical study dealing with the propagation properties of Love wave along multi-guide layers, and the effects on temperature stability of the thickness of the guide layers.

Table 1. Physical parameters of the materials for guide layer and substrate.

\begin{tabular}{|c|c|c|c|}
\hline Materials & Shear Velocity $(\mathrm{m} / \mathrm{s})$ & Density $\left(\mathrm{kg} / \mathrm{m}^{3}\right)$ & Polarity of Temperature Coefficient \\
\hline $\begin{array}{l}\text { 64efficien } \\
\text { 41 efficien } \\
3\end{array}$ & $\begin{array}{r}4,608 \\
4,792 \\
\end{array}$ & 4,700 & - \\
\hline 3600 ficien $_{3}$ & 4,202 & 7,450 & - \\
\hline $\begin{array}{l}\text { ST-90ic quartz } \\
\text { 36artquartz }\end{array}$ & $\begin{array}{l}5,060 \\
5,100\end{array}$ & 2,650 & + \\
\hline $\mathrm{SiO}_{2}$ & 2,850 & 2,650 & + \\
\hline PMMA & 1,100 & 1,140 & - \\
\hline SU-8 & 1,810 & 1,190 & - \\
\hline
\end{tabular}

The purpose of this paper is to describes the Love wave propagation along the $\mathrm{ST}-90^{\circ} \mathrm{X}$ quartz substrate using a multi-guide structure like $\mathrm{SiO}_{2} / \mathrm{SU}-8$, as shown in Figure 1. A SU-8 thin layer is proposed as guide layer, which exhibits low shear SH velocity, and benefits for the improvement of behavior and durability of a Love-type device when working as a viscosity sensor in liquid media [14,15]. 
A theoretical model was established to extract the optimal design parameters of the Love wave device with multi-guides by solving the coupled electromechanical field equation [11], and the temperature coefficient of frequency (Tcf) of such a Love wave structure was calculated theoretically. Then the theoretical results were confirmed by the experimental results, in which, a Love wave delay line with operation frequency of $174 \mathrm{MHz}$ and $\mathrm{SiO}_{2} / \mathrm{SU}-8$ guiding layer on ST- $90^{\circ} \mathrm{X}$ quartz substrate was fabricated. Very excellent temperature stability of $\sim 2.16 \mathrm{ppm}$ was observed from the fabricated Love wave device in case where $0.21 \mu \mathrm{m} \mathrm{SiO}_{2}$ and $0.8 \mu \mathrm{m} \mathrm{SU}-8$ were coated as the guide layers.

Figure 1. The schematic of the Love wave device with multi-guide layers of $\mathrm{SiO}_{2} / \mathrm{SU}-8$.

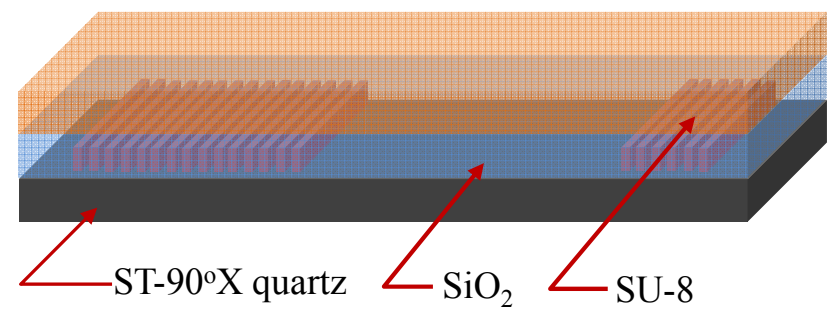

\section{Theoretical Analysis}

\subsection{Theory Model}

In this section, the temperature coefficient of frequency $(T c f)$ of a Love wave device with multi-guide layers was analyzed by solving the coupled electromechanical field equation combing with the approximate method described by Tomar et al. [11]. For the theoretical approach of the Love wave device, a layered structure composed of a semi-infinite piezoelectric substrate with IDT pattern, and multi-guide layers was constructed, as shown in Figure 2. In the present work the theoretical calculations on the layered structures is an application of the theory of elastic wave propagation developed by Farnell and Adler [16-18].

Figure 2. The coordination system in this study.

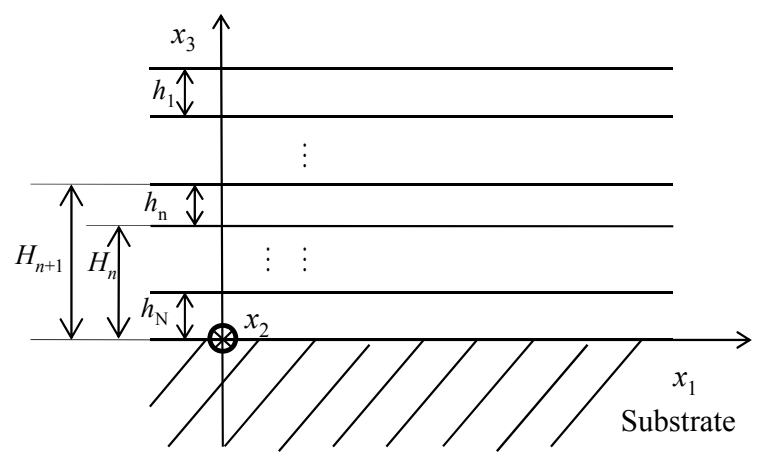

The coordinate system for the theoretical analysis of Love wave propagation along the layered structure is shown in Figure 2. All the parameters of the media are transformed into this coordinate system. It is well known that the constitutive equations in a piezoelectric medium are as follows: 


$$
\begin{aligned}
& \mathbf{T}=\mathbf{c}: \mathbf{S}-\mathbf{e}^{\prime} \cdot \mathbf{E} \\
& \mathbf{D}=\boldsymbol{\varepsilon} \cdot \mathbf{E}+\mathbf{e}: \mathbf{S}
\end{aligned}
$$

where $\mathbf{c}, \mathbf{e}$, and $\boldsymbol{\varepsilon}$ represent the elastic, piezoelectric, and permittivity constants matrices. $\mathbf{e}^{\prime}$ is the transposition of $\mathbf{e}$. The vectors $\mathbf{D}$ and $\mathbf{E}$ denote the electric displacement and the electric field intensity. The vectors $\mathbf{T}$ and $\mathbf{S}$ indicate the stress tensor and the strain tensor. The strain vector $\mathbf{S}$ can be expressed with the following term:

$$
\mathbf{S}=\nabla_{\mathrm{S}} \cdot \mathbf{u}
$$

where $\nabla_{\mathrm{S}}$ denotes the so-called symmetric gradient operator and the vector $\mathrm{u}$ denotes the particle displacement. The quasi-static approximation is adopted, where it is assumed that the electric field intensity can be described by the gradient of a scalar electric potential $\varphi$ :

$$
\mathbf{E}=-\nabla \varphi
$$

Equations (3) and (4) can be used to eliminate the vectors $\mathbf{S}$ and $\mathbf{E}$ from the constitutive Equations (1) and (2). Gravity and other initial stresses in the medium are neglected, thus the particle motion satisfies Newton's law:

$$
\nabla \cdot \mathbf{T}=\rho \frac{\partial^{2} \mathbf{u}}{\partial t^{2}}
$$

No external electric charges exist within the medium. Thus, the divergence of the electric displacement is zero:

$$
\nabla \cdot \mathbf{D}=0
$$

For the analysis situation mentioned above, the particle motion and the electric field in such a piezoelectric medium satisfy the following equations:

$$
\left\{\begin{array}{l}
c_{i j k l} \frac{\partial^{2} u_{k}}{\partial x_{j} \partial x_{l}}+e_{i j k} \frac{\partial^{2} \varphi}{\partial x_{i} \partial x_{k}}-\rho \frac{\partial^{2} u_{k}}{\partial t^{2}}=0 \\
e_{j k l} \frac{\partial^{2} u_{k}}{\partial x_{j} \partial x_{l}}-\varepsilon_{j k} \frac{\partial^{2} \varphi}{\partial x_{j} \partial x_{k}}=0
\end{array} i, j, k, l=1,2,3\right.
$$

where $\rho$ is the mass density of the considered material. Equation (7) describes the propagation of the acoustic wave and its associated electric field. The Love wave mode $\left(u_{1}, u_{3}\right)$ decoupling with $u_{2}$ and $\varphi$ while $u_{2}$ coupling with $\varphi$ is discussed in this paper.

(1) In the piezoelectric substrate:

The substrate in this paper is a piezoelectric medium whose particle motion in the $\mathrm{x}_{2}$-direction is coupled with the electric field. The general solution assumed within the substrate is:

$$
\left\{\begin{array}{l}
u_{2}=U \exp \left\{-i k\left(x_{1}+\beta x_{3}\right)\right\} \exp (i w t) \\
\varphi=\Phi \exp \left\{-i k\left(x_{1}+\beta x_{3}\right)\right\} \exp (i w t)
\end{array}\right.
$$

here $U$ and $\Phi$ are the magnitudes of the particle motion and the electric potential. $\beta$ is a deep attenuation factor; $\omega$ is an angular frequency; $\mathrm{k}=2 \pi / \lambda$ is a wave number and $\lambda$ is a wavelength; 
$i=\sqrt{-1}$ is an imaginary unit. In this paper, both $\mathrm{k}$ and $\omega$ are assumed to be positive, real numbers. Substitution of the general solution Equation (8) into Equation (7) produces a homogeneous algebraic set:

$$
\begin{aligned}
& {\left[\begin{array}{ll}
\Gamma_{11} & \Gamma_{12} \\
\Gamma_{12} & \Gamma_{22}
\end{array}\right] \times\left[\begin{array}{l}
U \\
\Phi
\end{array}\right]=0} \\
& \Gamma_{11}=c_{44} \beta^{2}+2 c_{46} \beta+c_{66}-\rho v^{2} \\
& \Gamma_{12}=e_{34} \beta^{2}+\left(e_{14}+e_{36}\right) \beta+e_{16} \\
& \Gamma_{22}=-\left(\varepsilon_{33} \beta^{2}+2 \varepsilon_{13} \beta+\varepsilon_{11}\right)
\end{aligned}
$$

where $v=\omega / k$ is the phase velocity of the acoustic waves. The determinant of the matrix of the coefficients of the set Equation (9) must vanish for nontrivial solutions, thus a quartic equation for $\beta$ is obtained. When $\mathrm{x}_{3} \rightarrow-\infty$, the particle displacement $u_{2}$ and the electric potential $\varphi$ must be zero, which means only the two roots with positive real parts are reasonable. The particular solutions for the acoustic wave and the electric field in the substrate are consequently in the following forms:

$$
\left\{\begin{array}{l}
u_{2}=\left[M_{1} A_{1} \exp \left(-i k \beta_{S 1} x_{3}\right)+M_{2} A_{2} \exp \left(-i k \beta_{S 2} x_{3}\right)\right] \cdot \exp \left\{i\left(\omega t-k x_{1}\right)\right\} \\
\varphi=\left[M_{1} \exp \left(-i k \beta_{S 1} x_{3}\right)+M_{2} \exp \left(-i k \beta_{S 2} x_{3}\right)\right] \cdot \exp \left\{i\left(\omega t-k x_{1}\right)\right\}
\end{array}\right.
$$

where $\mathrm{A}$ is the ratio of $\mathrm{U}$ to $\Phi$ and:

$$
\left\{\begin{array}{l}
A_{1}=\left.\frac{U}{\Phi}\right|_{\beta_{1}}=\frac{\varepsilon_{33} \beta_{S 1}^{2}+2 \varepsilon_{13} \beta_{S 1}+\varepsilon_{11}}{e_{34} \beta_{S 1}^{2}+\left(e_{14}+e_{36}\right) \beta_{S 1}+e_{16}} \\
A_{2}=\left.\frac{U}{\Phi}\right|_{\beta_{2}}=\frac{\varepsilon_{33} \beta_{S 2}^{2}+2 \varepsilon_{13} \beta_{S 2}+\varepsilon_{11}}{e_{34} \beta_{S 2}^{2}+\left(e_{14}+e_{36}\right) \beta_{S 2}+e_{16}}
\end{array}\right.
$$

The $\mathrm{M}$ coefficient in Equation (11) and in the following expressions is the coefficient to be determined by the boundary conditions.

(2) In the elastic wave-guide layer:

The particle displacement is uncoupled from the electric field within the layers because the layers are assumed to be isotropic materials without piezoelectricity. Suppose several layers were overlaid on the substrate, define the top layer the first one, the one above the substrate the $\mathrm{N}^{\text {th }}$. The layer is finite in the $\mathrm{x}_{3}$-direction and the solution for acoustic waves in the $\mathrm{n}^{\text {th }}$ layer is of the following form:

$$
u_{2 n}=\left[U_{n 1} \exp \left(-i k \beta_{n} x_{3}\right)+U_{n 2} \exp \left(i k \beta_{n} x_{3}\right)\right] \cdot \exp \left\{i\left(w t-k x_{1}\right)\right\} \quad H_{n+1}<x_{3}<H_{n}
$$

where $\beta_{\mathrm{n}}=\sqrt{v^{2} / V_{\mathrm{n}}^{2}-1}$ and $V_{n}=\sqrt{\mu_{n} / \rho_{n}}$ is the velocity of shear bulk acoustic wave in the waveguide material. $\mu_{n}$ and $\rho_{n}$ are the shear modulus and mass density of the $n^{\text {th }}(n=1-N)$ layer medium, respectively.

The electric potential in the $\mathrm{n}^{\text {th }}$ layer can be expressed by:

$$
\varphi_{\mathrm{n}}=\left[\Phi_{n 1} \exp \left(-k x_{3}\right)+\Phi_{n 2} \exp \left(k x_{3}\right)\right] \cdot \exp \left\{i\left(w t-k x_{1}\right)\right\} \quad H_{n+1}<x_{3}<H_{n}
$$


(3) In the air or vacuum

Only electric potential exists in the air or vacuum, and the electric potential will be zero when $\mathrm{x}_{3} \rightarrow-\infty$, thus the solution for the electric potential above the guide layer is of the following form:

$$
\varphi_{0}=\Phi_{0} \exp \left(-k x_{3}\right) \exp \left\{\mathrm{i}\left(\omega t-k x_{1}\right)\right\} \quad x_{3}>H_{1}
$$

Then, the values of the undetermined $M$ coefficients in Equation (10) are decided by the boundary conditions at the interface and the surface of the layered structure as following:

(1) Mechanical boundary conditions: the particle displacement and stress in the $\mathrm{x}_{3}$-direction are continuous on every interface. The normal stress is zero on the surface of the top layer.

(2) Electric boundary conditions: the electric potential and electric displacement in the $\mathrm{x}_{3}$-direction are continuous on every interface.

Hence, the dispersion equation of Love waves in a multi-waveguides structure can be obtained by the wave equations and the boundary conditions:

$$
\frac{T_{2}-X_{N} A_{2}}{T_{1}-X_{N} A_{1}}=\frac{D_{2}-i \overline{\varepsilon_{N}}}{D_{1}-i \overline{\varepsilon_{N}}}
$$

where:

$$
\begin{gathered}
X_{1}=-i \mu_{1} \beta_{1} \tan \left(k b_{1} h_{1}\right) \\
X_{n}=\mu_{n} \beta_{n} \frac{i \tan \left(k \beta_{n} h_{n}\right) \mu_{n} \beta_{n}+X_{n-1}}{i \tan \left(k \beta_{n} h_{n}\right) X_{n-1}+\mu_{n} \beta_{n}}, n=2,3, \cdots, N \\
\left\{\begin{array}{l}
T_{1}=\left(c_{44} \beta_{S 1}+c_{46}\right) A_{1}+\left(e_{34} \beta_{S 1}+e_{14}\right) \\
T_{2}=\left(c_{44} \beta_{S 2}+c_{46}\right) A_{2}+\left(e_{34} \beta_{S 2}+e_{14}\right)
\end{array}\right. \\
\left\{\begin{array}{l}
D_{1}=\left(e_{34} \beta_{S 1}+e_{36}\right) A_{1}-\left(\varepsilon_{33} \beta_{S 1}+\varepsilon_{31}\right) \\
D_{2}=\left(e_{34} \beta_{S 2}+e_{36}\right) A_{2}-\left(\varepsilon_{33} \beta_{S 2}+\varepsilon_{31}\right)
\end{array}\right. \\
\left\{\begin{array}{l}
\overline{\varepsilon_{1}}= \\
\varepsilon_{1} \frac{\varepsilon_{1} \tanh \left(k h_{1}\right)+\varepsilon_{0}}{\varepsilon_{0} \tanh \left(k h_{1}\right)+\varepsilon_{1}} \\
\overline{\varepsilon_{n}}=\varepsilon_{\mathrm{n}} \frac{\varepsilon_{\mathrm{n}} \tanh \left(k h_{n}\right)+\overline{\varepsilon_{n-1}}}{\overline{\varepsilon_{n-1}} \tanh \left(k h_{n}\right)+\varepsilon_{\mathrm{n}}}
\end{array}\right.
\end{gathered}
$$

It is well-known that the material constants of the substrate and the layers vary with different temperature. Thus, the temperature-dependent Love wave phase velocity can be calculated using Equation (15) with temperature dependent material constants. The temperature coefficient of frequency $(T c f)$ can be defined as:

$$
T c f=\frac{v_{35}-v_{15}}{20 v_{25}}-\alpha
$$

where $\alpha$ is coefficient of thermal expansion in the substrate, $v_{15}, v_{25}, v_{35}$ refer to Love wave phase velocity at $15{ }^{\circ} \mathrm{C}, 25{ }^{\circ} \mathrm{C}, 35^{\circ} \mathrm{C}$. 
The temperature dependent material constants is approximated by a second-order function:

$$
X=X_{0}\left[1+\alpha_{1}\left(T-T_{0}\right)+\alpha_{2}\left(T-T_{0}\right)^{2}\right]
$$

where $X$ refers to material constants at various temperature of $T, X_{0}$ is the material constants at room temperature, $T_{0}, \alpha_{1}$ and $\alpha_{2}$ are the first- and second-order temperature coefficients of the material constants, respectively. Table 2 lists the material constants used in this paper.

Table 2. The temperature-dependent parameters of the substrate and guide layers $[11,19,20]$.

\begin{tabular}{|c|c|c|c|c|}
\hline Materials & Parameters & $\begin{array}{c}X_{0} \text { at reference } \\
\text { temperature of } T_{0}\end{array}$ & $\begin{array}{c}\alpha_{1} \\
\left(10^{-4}\right)\end{array}$ & $\begin{array}{c}\alpha_{2} \\
\left(10^{-7}\right)\end{array}$ \\
\hline \multirow{14}{*}{$\begin{array}{c}\text { Quartz } \\
\mathrm{T}_{0}=20^{\circ} \mathrm{C}\end{array}$} & $\mathrm{c}_{11}\left(10^{11} \mathrm{~N} / \mathrm{m}^{2}\right)$ & 0.8674 & -0.443 & -4.07 \\
\hline & $c_{12}\left(10^{11} \mathrm{~N} / \mathrm{m}^{2}\right)$ & 0.0699 & -29.3 & 72.45 \\
\hline & $c_{13}\left(10^{11} \mathrm{~N} / \mathrm{m}^{2}\right)$ & 0.1191 & -4.92 & -5.96 \\
\hline & $c_{14}\left(10^{11} \mathrm{~N} / \mathrm{m}^{2}\right)$ & -0.1791 & 0.98 & -0.13 \\
\hline & $c_{33}\left(10^{11} \mathrm{~N} / \mathrm{m}^{2}\right)$ & 1.072 & -1.88 & -14.12 \\
\hline & $\mathrm{c}_{44}\left(10^{11} \mathrm{~N} / \mathrm{m}^{2}\right)$ & 0.5794 & -1.72 & -2.25 \\
\hline & $\mathrm{c}_{66}\left(10^{11} \mathrm{~N} / \mathrm{m}^{2}\right)$ & 0.3988 & 1.8 & 2.01 \\
\hline & $\rho\left(10^{3} \mathrm{~kg} / \mathrm{m}^{3}\right)$ & 2.65 & -0.3492 & -0.159 \\
\hline & $\mathrm{e}_{11}\left(\mathrm{C} / \mathrm{m}^{2}\right)$ & 0.171 & & \\
\hline & $\mathrm{e}_{14}\left(\mathrm{C} / \mathrm{m}^{2}\right)$ & 0.0403 & & \\
\hline & $\varepsilon_{11}\left(10^{-11} \mathrm{~F} / \mathrm{m}^{2}\right)$ & 3.997 & & \\
\hline & $\varepsilon_{33}\left(10^{-11} \mathrm{~F} / \mathrm{m}^{2}\right)$ & 4.103 & & \\
\hline & $\alpha_{11}\left(10^{-6} /{ }^{\circ} \mathrm{C}\right)$ & 13.71 & & \\
\hline & $\alpha_{33}\left(10^{-6} /{ }^{\circ} \mathrm{C}\right)$ & 7.48 & & \\
\hline \multirow{3}{*}{$\begin{array}{c}\mathrm{SiO}_{2} \\
\mathrm{~T}_{0}=25^{\circ} \mathrm{C}\end{array}$} & $\rho\left(10^{3} \mathrm{~kg} / \mathrm{m}^{3}\right)$ & 2.2 & & \\
\hline & $\mu\left(10^{11} \mathrm{~N} / \mathrm{m}^{2}\right)$ & 0.3121 & 1.4553 & \\
\hline & $\varepsilon_{11}\left(10^{-11} \mathrm{~F} / \mathrm{m}^{2}\right)$ & 3.8265 & 0.2628 & \\
\hline \multirow{3}{*}{$\begin{array}{c}\mathrm{SU}-8 \\
\mathrm{~T}_{0}=25^{\circ} \mathrm{C}\end{array}$} & $\rho\left(10^{3} \mathrm{~kg} / \mathrm{m}^{3}\right)$ & 1.2152 & -4.5142 & 1.3302 \\
\hline & $\mu\left(10^{11} \mathrm{~N} / \mathrm{m}^{2}\right)$ & 0.011769 & -37.757 & 83.3139 \\
\hline & $\varepsilon_{11}\left(10^{-11} \mathrm{~F} / \mathrm{m}^{2}\right)$ & 2.6563 & & \\
\hline
\end{tabular}

\subsection{Numerical Results and Discussion}

Here, we just consider the Love wave propagating along the multi-guiding layer on ST- $90^{\circ} \mathrm{X}$ quartz, and the $\mathrm{SiO}_{2} / \mathrm{SU}-8$ act as the wave guide layers, and the temperature-dependent mechanical parameters of the guiding layers and substrates like the density, elastic constants, piezoelectricity and dielectric constants of the above media are as listed in Table 2.

Based on the theoretical formulas mentioned above, and the mechanical parameters of the substrate and guide layers listed in Table 2, the dispersion properties of a Love wave in a layered structure can be analyzed, and the phase velocity of the Love wave depending on the guiding layer thickness at room temperature of $25^{\circ} \mathrm{C}$ is as depicted in Figure 3. Only the fundamental Love wave mode exists under the thickness of waveguides we are interested in. From the calculated results, the Love wave velocity decreases with increases of the thickness of the guide layers, and the effect on the Love wave velocity from the SU-8 thickness was more obvious over the $\mathrm{SiO}_{2}$ guide layer. 
Figure 3. Phase velocity versus normalized layer thickness in a layered structure of $\mathrm{ST}-90^{\circ} \mathrm{X}$ quartz $/ \mathrm{SiO}_{2} / \mathrm{SU}-8$ at room temperature of $25^{\circ} \mathrm{C}$.

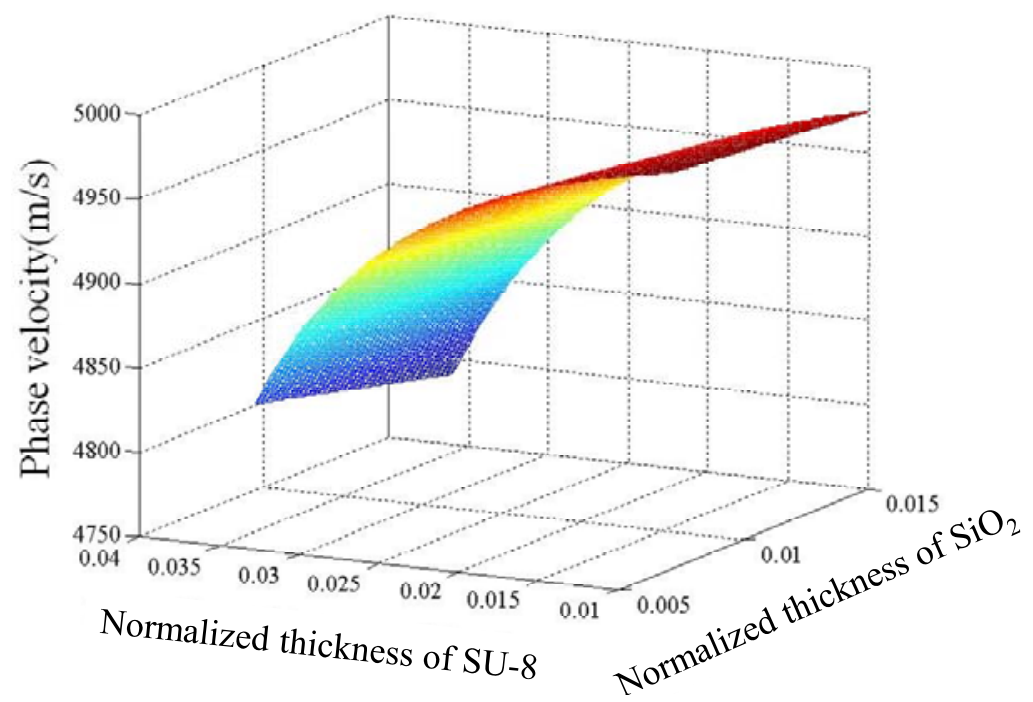

Figure 4 shows calculated $T c f$ versus normalized thickness of the guide layers in a layered structure of ST- $90^{\circ} \mathrm{X}$ quartz/SiO$/ 2 / \mathrm{SU}-8$ using Equation (20). The $T c f$ value is influenced by SU-8 thickness far more significantly than by that of $\mathrm{SiO}_{2}$. Considering the technical difficulties of thick $\mathrm{SiO}_{2}$ film deposition, a thin $\mathrm{SiO}_{2}$ coating is advised, so, from Figure 3, we can see that when the thickness of $\mathrm{SiO}_{2}$ is $0.2 \mu \mathrm{m}$ and SU-8 $0.826 \mu \mathrm{m}$ are applied; almost zero $T c f$ of the device may be achieved.

Figure 4. Calculated Tcf versus normalized layer thickness.

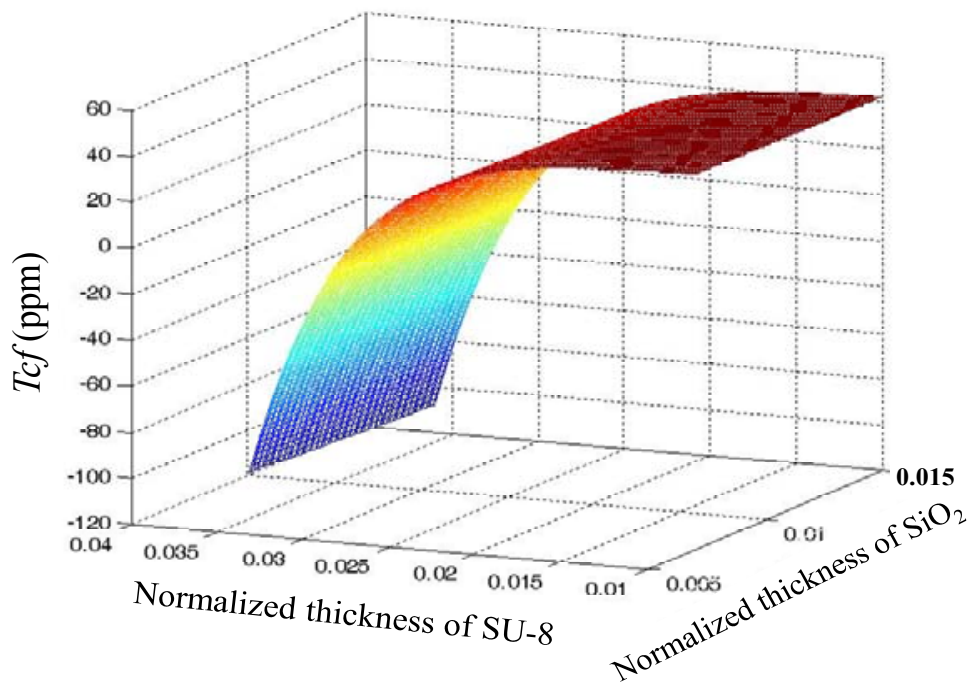

\section{Experiments}

\subsection{Fabrication of the Love Wave Device}

The Love wave delay lines with a $\mathrm{SiO}_{2} / \mathrm{SU}-8$ guiding layer were developed on a $\mathrm{ST}-90^{\circ} \mathrm{X}$ quartz substrate. First the SH-SAW delay line on ST- $90^{\circ} \mathrm{X}$ quartz with two photolithographically defined Al $(250 \mathrm{~nm})$ transducers separated by a path length (transducer center separation) of $2.5 \mathrm{~mm}$ was fabricated. The two transducers consist of 120 and 40 finger-pairs interdigitated electrodes, with 
periodicity of $28 \mu \mathrm{m}$ for an operation frequency of $175 \mathrm{MHz}$. A single phase unidirectional transducer (SPUDT) was used to structure the transducers to reduce the low insertion loss [21]. Then, $\mathrm{SiO}_{2}$ thin film was evaporated on the fresh surface of the SH-SAW device by the lift-off technique at room temperature with a sacrificial layer of photoresist 5214. The thickness of $\mathrm{SiO}_{2}$ was set to $0.21 \mu \mathrm{m}$ according to the theoretical prediction, and monitored by an Alpha-Step IQ profiler. After the $\mathrm{SiO}_{2}$ coating, the SU-8 2050 with various thicknesses produced by the Microchem Company was deposited onto the $\mathrm{SiO}_{2}$ surface by spin coating. The design parameters of the fabricated devices are as listed in Table 3 .

Table 3. Design parameters of the fabricated devices.

\begin{tabular}{|c|c|c|}
\hline Device \# & $\mathbf{S i O}_{2}$ Thickness $(\boldsymbol{\mu m})$ & SU-8 \\
\hline 1 & 0.21 & $0.9 / 0.032$ \\
2 & 0.21 & $0.1 / 0.0035$ \\
3 & 0.21 & $0.8 / 0.028$ \\
\hline
\end{tabular}

Figure 5(a) shows the fabricated Love wave devices with size of $9 \times 3 \mathrm{~mm}$, and also, the frequency response $\left(\mathrm{S}_{21}\right)$ of the fabricated Love wave device with SU-8 thickness of $0.8 \mu \mathrm{m}$, tagged as device \#1, which was characterized by a network analyzer (Advantest R3765), as shown in Figure 5(b). Insertion loss of $\sim 17 \mathrm{~dB}$ was observed at the frequency of $173.38 \mathrm{MHz}$.

Figure 5. (a) the fabricated Love wave device; (b) the measured frequency response $\left(\mathrm{S}_{21}\right)$ of the fabricated device.

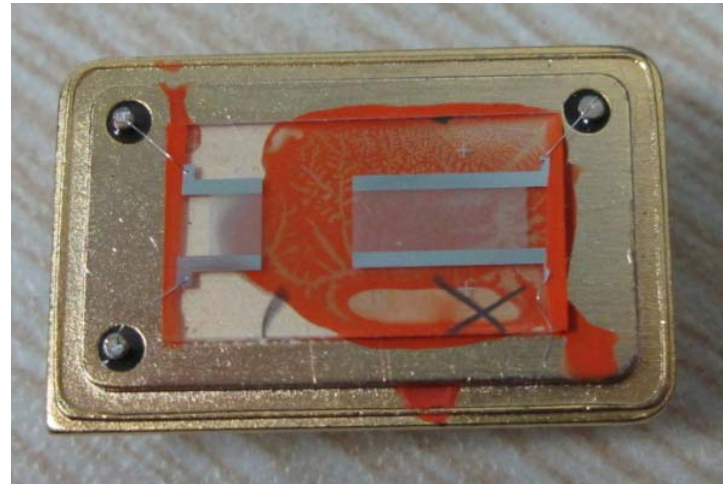

(a)

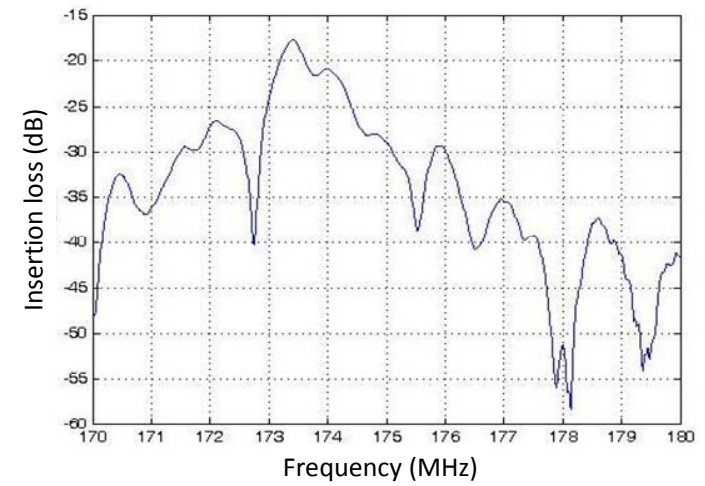

(b)

\subsection{Experimental Setup}

The temperature properties of the fabricated Love wave devices were evaluated experimentally. The measurement setup, which includes the network analyzer, temperature sensor, ceramic heating element and computer, is depicted in Figure 6. The fabricated Love wave delay lines with different SU-8 thicknesses listed in Table 2 were heated by the ceramic heating element, and the network analyzer was used to monitor and recording the frequency shift depending on the applied temperature change. The temperature change of the Love wave device is also read out by the temperature sensor connected to pt100 posted at the bottom of the Love wave device. Both frequency and temperature are acquired in real time by the PC connected to network analyzer and temperature sensor. 
Figure 6. The temperature stability measurement setup of the fabricated devices.

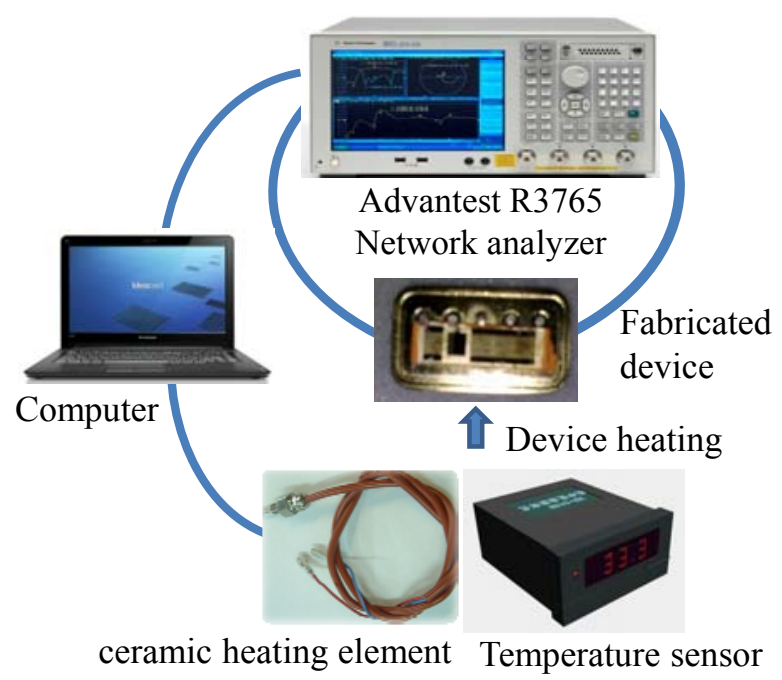

\subsection{Experimental Results and Discussion}

Prior to measurement, the baseline noise of the Love wave device in testing time of $60 \mathrm{~s}$ was tested at room temperature $\left(25^{\circ} \mathrm{C}\right)$. It is shown as the dotted line in Figure 7. A frequency shift of $\sim 10 \mathrm{kHz}$ was observed.

Figure 7. The measured baseline noise and time-dependent frequency response of the device \#3.

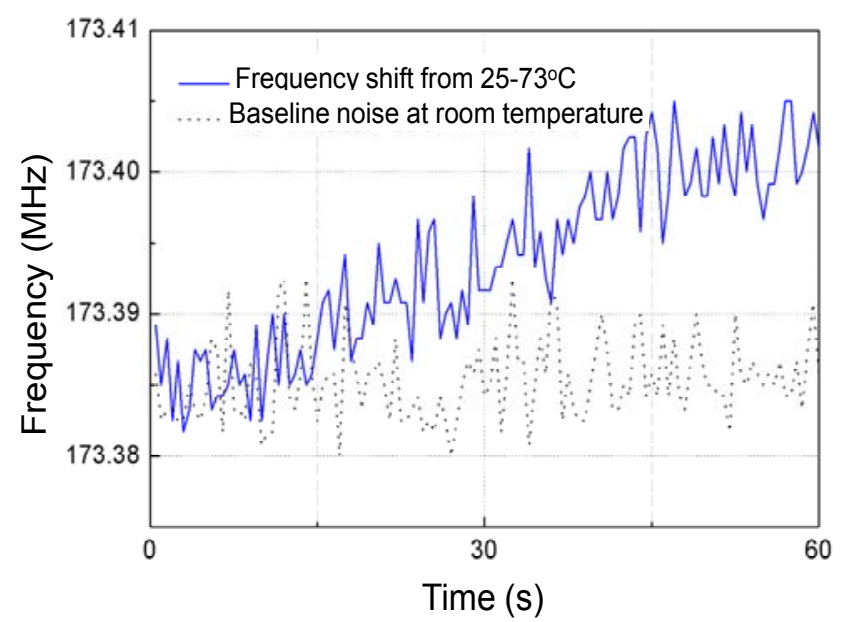

Then, the frequency response depending on temperature variation from $25{ }^{\circ} \mathrm{C}$ to $73{ }^{\circ} \mathrm{C}$ of the fabricated Love wave delay line (device \#3) was measured as shown in Figure 7, and a frequency shift of around $0.018 \mathrm{MHz}$ with a temperature increase of $48{ }^{\circ} \mathrm{C}$ was observed. The binomial fitting of the measured frequency shift in temperature range of $25^{\circ} \mathrm{C} \sim 73{ }^{\circ} \mathrm{C}$ was plotted in Figure 8. A very low $T c f$ of $2.16 \mathrm{ppm} /{ }^{\circ} \mathrm{C}\left(T c f=0.018 / 48 / 173.38 \times 10^{6}\right)$ was deduced experimentally, and it is far more less than typical Love wave structure $\left(\mathrm{ST}-90^{\circ} \mathrm{X}\right.$ quartz $\left./ \mathrm{SiO}_{2}\right)$ of $27-31 \mathrm{ppm} /{ }^{\circ} \mathrm{C}$ [8]. Also, due to the insertion loss effect due to strain, and the stress effect between the $\mathrm{SU}-8$ and $\mathrm{SiO}_{2}$ layers as temperature increases, another insertion loss of $\sim 5 \mathrm{~dB}$ was induced when the temperature was increased to $73{ }^{\circ} \mathrm{C}$. The temperature properties of another two devices (device $1 \#$ and $2 \#$ ) in the temperature range of 
$25{ }^{\circ} \mathrm{C} \sim 73{ }^{\circ} \mathrm{C}$ were also tested as shown in Figure 9, and the corresponding Tcf values for the device 1\# and 2\# sre depicted in Figure 10.

Figure 8. The time-dependent frequency response of the device \#3.

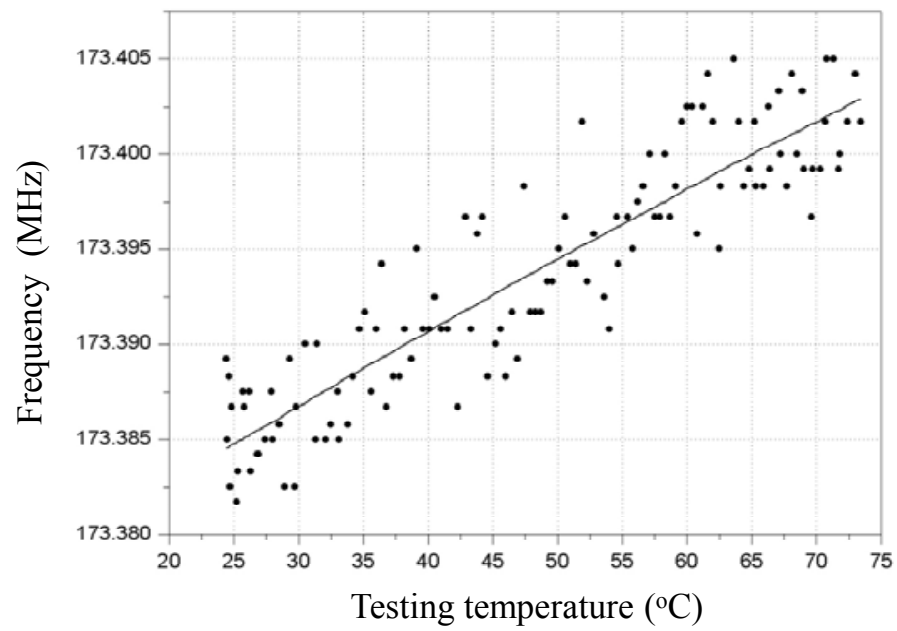

Figure 9. Measured temperature-dependent frequency shift of the devices \#1, \#2, and \#3.

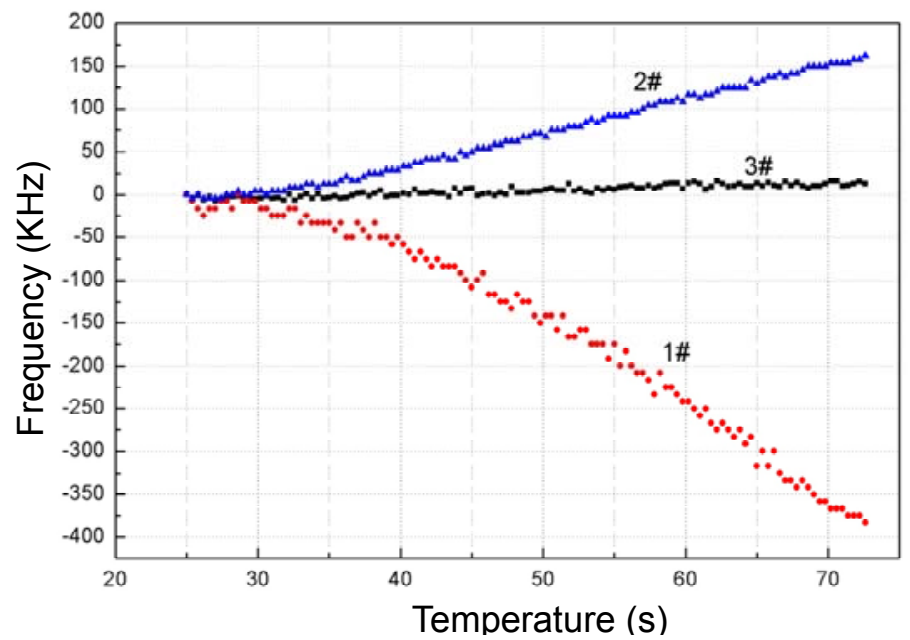

Figure 10. $T c f$ comparison between the experimental results and theoretical calculations.

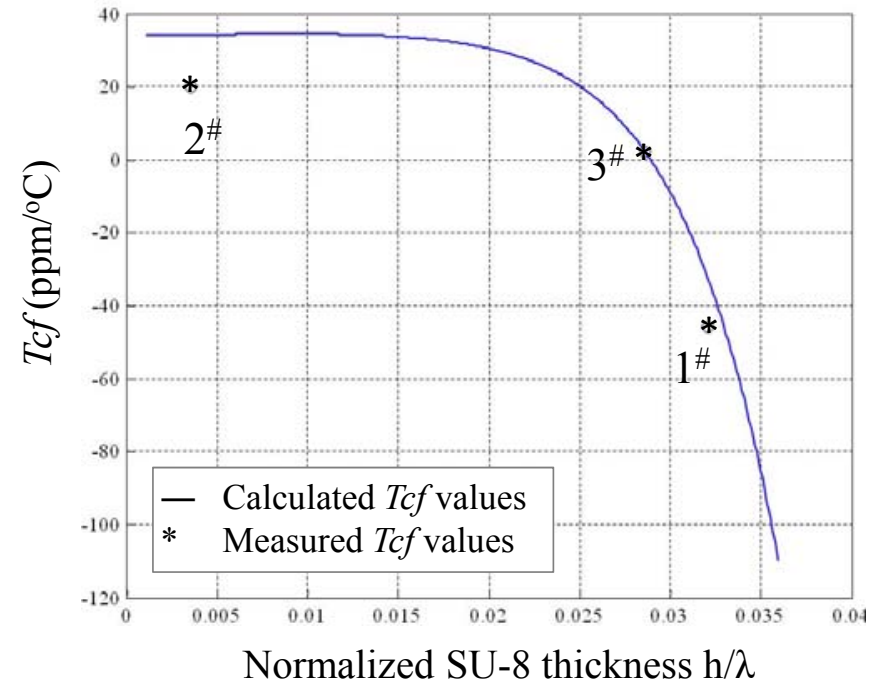


The measured data agrees well with the theoretical analysis as above. When thicker SU-8 was applied, the frequency decreases quickly due to the larger viscoelastic nature of the polymer itself. It means that the optimal thicknesses of the multi-guide layers of $\mathrm{SiO}_{2} / \mathrm{SU}-8$ are determined as $0.21 \mu \mathrm{m} / 0.8 \mu \mathrm{m}$. From these promising results, we conclude that the temperature stability of a Love wave device can be improved effectively by controlling the wave guide layer thickness.

\section{Conclusions}

The temperature effect on Love wave propagation along a layered structure was analyzed theoretically. The Love wave device with multi-guide layers of $\mathrm{SU}-8 / \mathrm{SiO}_{2} / \mathrm{ST}-90^{\circ} \mathrm{X}$ quartz and a very low temperature coefficient of frequency $(T c f)$ of $2.16 \mathrm{ppm} /{ }^{\circ} \mathrm{C}$ was implemented based on establishment of the corresponding theoretical model. The optimal guide layer thicknesses were determined, leading to excellent temperature stability. The theoretical model was confirmed by the experiments.

\section{Acknowledgments}

The authors gratefully acknowledge the support of the National Natural Science Foundation of China: No. 11074268 and 10974171.

\section{References}

1. Gizeli, E. Design considerations for the acoustic waveguide biosensor. Smart Mater. Struct. 1997, 6, 700-706.

2. Kovacs, G.; Vellekoop, M.J.; Haueis, R. A love wave sensor for (bio)chemical sensing in liquids. Sens. Actuators A 1994, 43, 38-43.

3. Gizeli, E.; Bender, F.; Rasmusson, A. Sensitivity of the acoustic wave guide biosensor to protein binding as a function of thewaveguide properties. Biosens. Bioelectron. 2003, 18, 1399-1406.

4. Zimmermann, C.; Rediere, D.; Dejous, C. A love-wave gas sensor coated with functionalized polysiloxane for sensing organophosphorus compounds. Sens. Actuators B 2001, 76, 86-94.

5. Jakoby, B.; Ismail, G.M.; Byfield, M.P. A novel molecularly imprinted thin film applied to a Love wave gas sensor. Sens. Actuators A 1999, 76, 93-97.

6. Bender, F.; Cernosek, R.W.; Josse, F. Love-wave biosensors using cross-linked polymer wave guides on LiTaO3 substrates. Electron. Lett. 2000, 36, 1672-1673.

7. Wang, W.; He, S. Theoretical analysis on response mechanism of polymer-coated chemical sensor based Love wave in viscoelastic media. Sens. Actuators B 2009, 138, 432-440.

8. Du, J.; Harding, G.L.; Ogilvy, J.A. A study of Love-wave acoustic sensors. Sens. Actuators A 1996, 56, 211-219.

9. Du, J.; Harding, G.L. A multilayer structure for Love-mode acoustic sensors. Sens. Actuators A 1998, 65, 152-159.

10. Hickernell, F.S.; Knuth, H.D.; Dablemont, R.C. The Surface Acoustic Wave Propagation Characteristics of $41^{\circ}$ Lithium Niobate with Thin Film $\mathrm{SiO}_{2}$. In Proceeding of IEEE International Frequency Control Symposium, Honolulu, HI, USA, 5-7 June 1996; pp. 216-221. 
11. Tomar, M.; Gupta, V.; Mansingh, A. Temperature stability of $c$-axis oriented $\mathrm{LiNbO}_{3} / \mathrm{SiO}_{2} / \mathrm{Si}$ thin film layered structures. J. Phys. D: Appl. Phys. 2001, 34, 2267-2273.

12. Tomar, M.; Gupta, V.; Sreenivas, K. Temperature coefficient of elastic constants of $\mathrm{SiO}_{2}$ over-layer on LiNbO3 for a temperature stable SAW device. J. Phys. D: Appl. Phys. 2003, 36, 1773-1777.

13. Parker, T.E.; Schulz, M.B. $\mathrm{SiO}_{2}$ film overlays for temperature-stable surface acoustic wave devices. Appl. Phys. Lett. 1975, 26, 75-77.

14. Roach, P.; Atherton, S.; Doy, N. SU-8 guiding layer for love wave devices. Sensors 2007, 7, 2539-2547.

15. Lee, S.; Kim, K.-B.; Kim, Y., II. Love wave SAW biosensors for detection of antigen-antibody binding and comparison with SPR biosensor. Food Sci. Biotechnol. 2011, 20, 1413-1418.

16. Auld, B.A. Acoustic Resonators. In Acoustic Fields and Waves in Solids, 2nd ed.; John Wiley \& Sons, Inc.: New York, NY, USA, 1973; Volume 1, pp. 265-348.

17. Farnell. G.W. Symmetry Considerations for Elastic Layer Modes Propagating in Anisotropic piezoelectric Crystal. IEEE Trans. Sonics Ultrason. 1970, 17, 229-238.

18. Liu, J.S.; He, S.T. Properties of Love waves in layered piezoelectric structures. Int. J. Solids Struct. 2010, 47, 169-174.

19. Zhang, G.W.; Shi, W.K.; Ji, X.J. Temperature Characteristics of Surface Acoustic Waves Propagating on $\mathrm{La}_{3} \mathrm{Ga}_{5} \mathrm{SiO}_{4}$ Substrates. J. Mater. Sci. Technol. 2004, 20, 63-66.

20. MPDB software. Material Property Database [DB/OL]. Available online: http://www.jahm.com/ (accessed on 24 May 2012).

21. Wang, W.; He, S.T.; Li, S.Z. Enhanced sensitivity of SAW gas sensor coated molecularly imprinted polymer incorporating high frequency stability oscillator. Sens. Actuators B 2007, 125, $422-427$.

(C) 2012 by the authors; licensee MDPI, Basel, Switzerland. This article is an open access article distributed under the terms and conditions of the Creative Commons Attribution license (http://creativecommons.org/licenses/by/3.0/). 